\title{
Narrow-band reflector coatings: design procedures
}

\author{
Hrvoje Zorc, Vesna Janicki, and Antun Peršin
}

\begin{abstract}
A class of problem dealing with narrow-band reflectors under oblique light incidence has been selected, and a group of design procedures leading to satisfactory results is analyzed. Initially performance criteria and design restrictions are set. Various designs are then created and analyzed. Conclusions can then be drawn on the relative merits of the designs. Two different types of target function have been chosen. A global search for 50 or more layers with varied optical thicknesses between 0 and $\lambda / 2$ and a needle design method have been applied to the synthesis. Gradient and variable metrics have been used for further refinement. Results are compared, and it is shown that all the design methods used yield similar results for this problem. The issue of how to determine the best method is addressed. (c) 1998 Optical Society of America

OCIS codes: $230.4170,310.0310,310.1620,230.1360$.
\end{abstract}

\section{Introduction}

Sophisticated interference coatings have been introduced into many applications in optics and optoelectronics. The demand for such coatings is a new challenge to designers. Often these demands are not purely scientific considerations but also demands restricted by the available technology and time. Designers are often faced with demanding requests from customers. Even worse, there may be no appropriate solutions already available (no procedures in handbooks), so they must look for an original solution, which takes time and increases product price.

For these problems selecting a suitable starting design is not easy. Here thin-film synthesis methods can be quite effective because they do not require a starting design, or if they do, they generate their own starting design automatically. The synthesis is then followed by a refinement method. A good comparison of the different refinement methods and their effectiveness has been presented by Dobrowolski and Kemp. ${ }^{1}$ We checked some of the refinement methods and noted that, in the sense of finding the minimum, they work in a similar way. Thus full attention is given to the synthesis strategy.

In this paper we define a case study for analyzing

The authors are with Ruđer Bošković Institute, Bijenička c. 54, P.O. Box 1016, 10001 Zagreb, Croatia. H. Zorc is also with Omega Optical Inc., P.O. Box 573, Brattleboro, Vermont 05302.

Received 4 December 1997; revised manuscript received 10 February 1998.

0003-6935/98/194249-05\$15.00/0

(C) 1998 Optical Society of America the efficiency of some synthesis methods and for determining the best strategy. Two synthesis methods have been chosen for analysis and comparison: global synthesis, sometimes called also the comprehensive search, ${ }^{2,3}$ and Tikhonravov's needle design method.4,5 Gradient and variable metrics have been used for refinement. In Refs. 6-8 the effect of the needle design method on some different types of coatings is described, but in this paper we attempt to analyze how the chosen methods and the strategy of their application have led to solutions. Solutions are different, and here we try to select the best or the most typical one for the chosen case study. We performed all calculations using TFCalc software. ${ }^{9}$

\section{Case Study and Synthesis Procedures}

We have chosen a type of problem dealing with narrow-band reflectors under oblique incidence. Initially goals and restrictions are defined. We then analyzed and compared the various designs. We impose the following requirements at oblique incidence (45 deg):

(A) Reflectance, $<1 \%$ for wavelengths of $420-530$ $\mathrm{nm}$.

(B) Reflectance, $>99 \%$ for wavelengths of $530-570$ $\mathrm{nm}$.

(C) Reflectance, $<1 \%$ for wavelengths of $570-600$ nm.

(D) Reflectance, $>99 \%$ for wavelengths of $600-650$ $\mathrm{nm}$.

(E) Reflectance, $<1 \%$ for wavelengths of $650-750$ $\mathrm{nm}$. 
Reflectance is the average reflectance $-R=$ $\left(R_{s}+R_{p}\right) / 2$. The refractive indices used for the design were as follows: $H, n=2.35, k=5 \times 10^{-4}$; $L, n=1.46, k=0$. The refractive indices were not dispersed. The incident medium was air, and the substrate material was BK7 optical glass. The number of layers should not exceed 50, and the minimum physical thickness of a layer was restricted to $10 \mathrm{~nm}$. The maximum thickness was restricted to $300 \mathrm{~nm}$.

The multilayer synthesis and refinement have been performed by use of two target functions: a discrete target function consisting of 226 points spaced $2 \mathrm{~nm}$ apart and a continuous target function in which requirements $(\mathrm{A})-(\mathrm{E})$ were entered as five continuous optimization targets. The target functions have been constructed following these requirements. The reflectance bands had half of the tolerance of the transmittance bands.

Optical thicknesses in the global search synthesis have been varied between 0 and $\lambda / 2$. For all design strategies $\lambda$ was $550 \mathrm{~nm}$. It actually has no special meaning, because the final results are physical thicknesses, but $\lambda$ has been given only to simplify the descriptions of some of the starting designs. A wider range of starting optical thicknesses was analyzed as well $(0-\lambda)$ but, surprisingly, with no improvements! It was expected that this approach might yield additional good designs owing to the half-order phenomena. It seems that this starting range has been restricted too much to reach them and still achieve better results.

Since this research is very basic, full attention is given to determining the most suitable strategy and not to the time it will require. All calculations were performed on a $166-\mathrm{MHz}$ personal computer. Several stopping criteria during minimization were applied, i.e., deviation of the obtained spectra from the target value $(\Delta)$, the number of layers, the total thickness. Designing on the best final design was not easy. The greater the number of parameters chosen, the more difficult it is to determine the best design. Tikhonravov recently discussed the dilemma of choosing the "best" design. ${ }^{10}$

\section{A. Global Search}

We started the global search from 50 alternating layers, where thicknesses were set at $\lambda / 4$ initially. Random variations of layer thicknesses were then performed. All generated thicknesses were in the range between 0 and $\lambda / 2$. Typically 1000 designs have been generated, in which $5-10$ of the best were chosen for further refinement. Later we note that the generation of multilayers starting with 70-75 layers yielded better results because of the decrease in the number of layers during the refinement procedure. Thus the final design fell into the given frames. The five best results of that design strategy are presented in Fig. 1.

\section{B. Modified Global Search}

This procedure enables some layers to have more freedom than others. Therefore we call the proce-

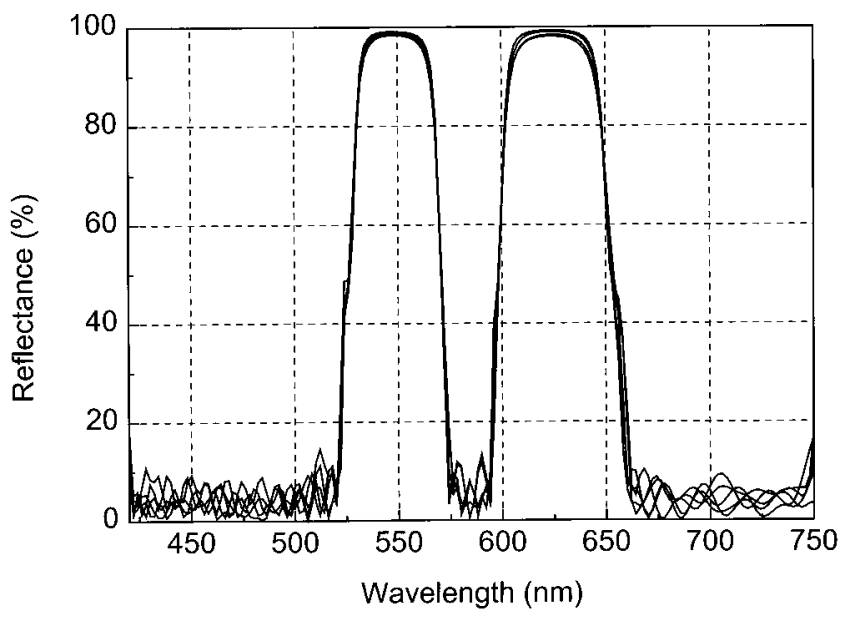

Fig. 1. Reflectance versus wavelength (nanometers) for the filter obtained from the global search.

dure modified. Namely, we gave a kind of direction to the global search, using what could be called a pseudomulticavity bandpass filter. So, for the initial design for the global search, a six-cavity narrow-band filter-(HLHL2HLHLHL) ${ }^{6}$ - centered at $585 \mathrm{~nm}$, allowing $100 \%$ variations in thicknesses, was chosen at the start, and then the global search was performed. $\mathrm{H}$ and $\mathrm{L}$ are high- and low-index materials as mentioned above. More about multiple-cavity filters can be found in Ref. 11. In this way the spacer layers are favored, i.e., they obtained a higher level of freedom. In other words, the shape of the multidimensional space was significantly changed. We hoped that this approach would enable the minima regions to be found more easily and efficiently. In Fig. 2 we present the best results from using the modified global search method followed by a final refinement.

\section{Needle Design}

The needle design started from a single $\mathrm{H}$ layer. The optical thickness of the starting layer was varied between $7 \lambda$ and $20 \lambda$, where $\lambda$ was again $550 \mathrm{~nm}$. A

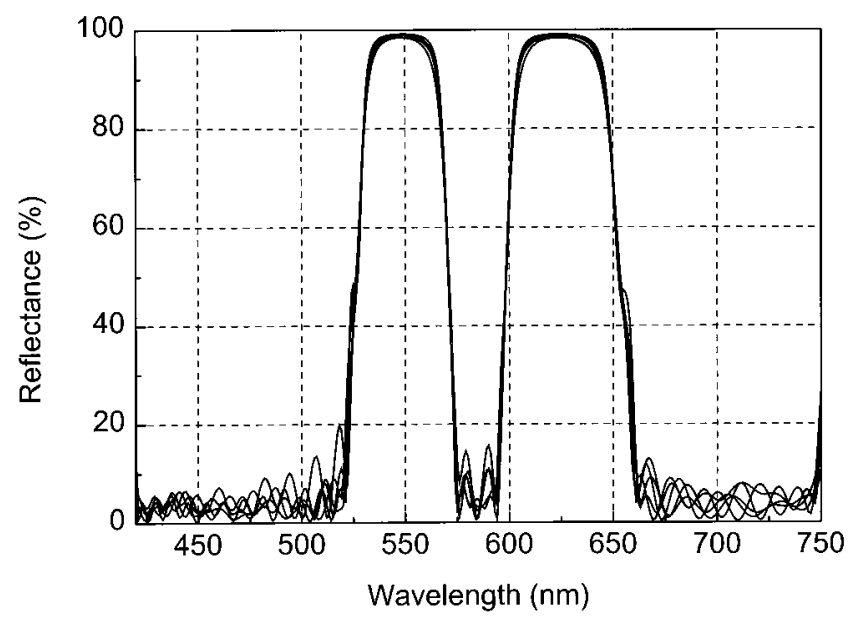

Fig. 2. Reflectance versus wavelength (nanometers) for the filter obtained from the modified global search. 


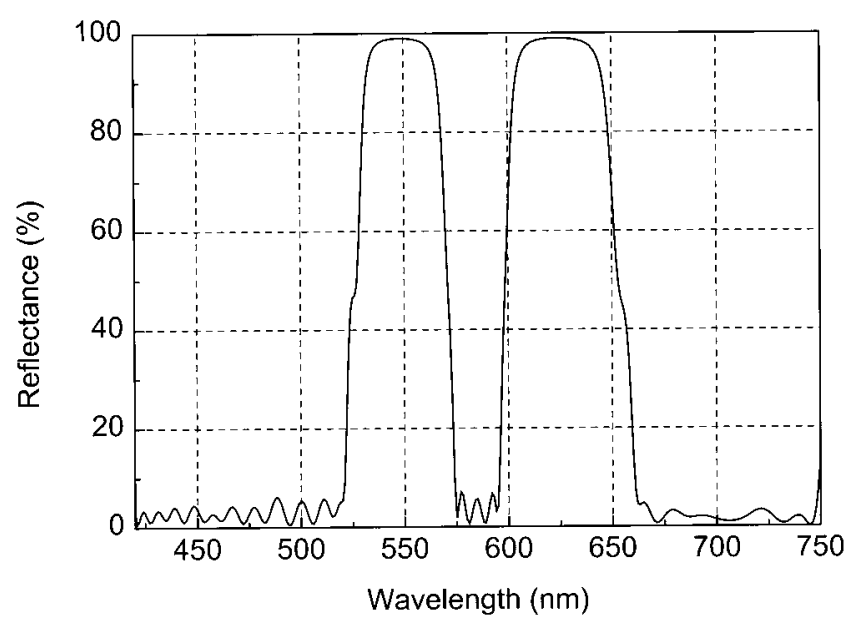

Fig. 3. Reflectance versus wavelength (nanometers) for the filter obtained from the needle method and the simultaneous insertion of layers.

thicker starting layer yielded more layers in the multilayer. The best result was obtained with a starting thickness of 20 $\lambda$. Larger starting thicknesses yielded too many layers, and their contribution to the performance was insignificant. Layers were added simultaneously and step by step. The step-by-step needle addition of layers enabled better control of the number of layers but yielded slightly greater deviations. The best results were obtained with a design process consisting of three or four simultaneous needle procedures, followed by refinement. The optimization of layers, which temporarily had zero thickness, has not stopped, but they are able to develop again. This approach actually increases the design time but yields better results. In other words, we did not artificially change the dimensions of the hyperspace, keeping the process going to the end as the needle method predicted.

The spectral characteristics of the filters, obtained by the above-mentioned needle design methods, are presented in Figs. 3 and 4 . Figure 3 corresponds to the needle design with the simultaneous insertion of layers and Fig. 4 to the step-by-step addition.

\section{Needle-Composite Design}

Two notch filters (centered at 550 and $625 \mathrm{~nm}$ ) have been designed with the needle design and some refinement procedures. The starting thicknesses of a high-index layer for the two needle designs are 10 $\lambda$. The first notch filter, having a high reflectance of $530-570 \mathrm{~nm}$, has 39 layers, and the second, having a high reflectance of $600-650 \mathrm{~nm}$, has 41 layers. Two notch filters were assembled through a simple L transition layer, and then the final variable metric refinement was performed. This approach has been found to give very good results in a relatively short time. The results of the described method are in Fig. 5.

\section{E. Composition of Two Narrow-Band Reflectors}

In the fifth method we composed the filter of two narrow-band reflectors of the $(\mathrm{L} 3 \mathrm{H})^{n}$ type followed by

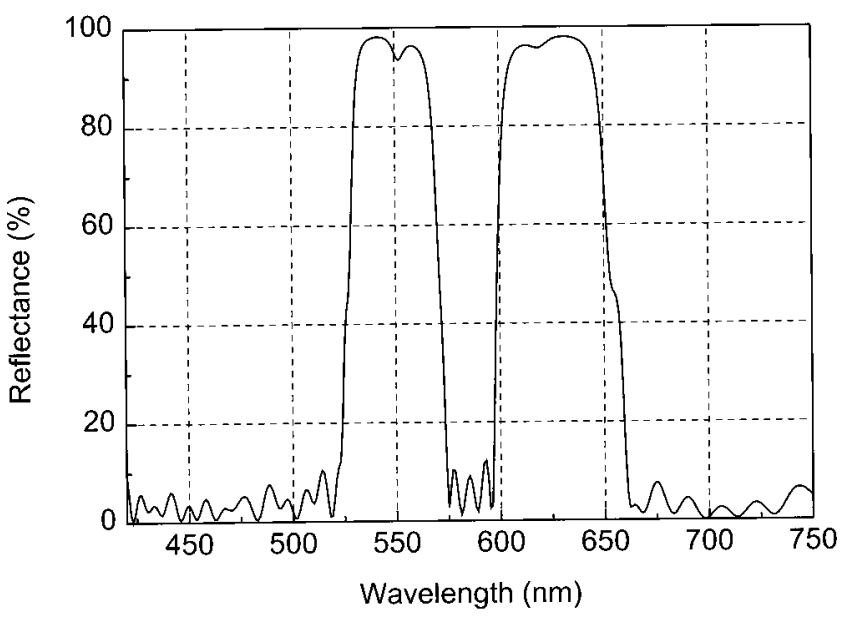

Fig. 4. Reflectance versus wavelength (nanometers) for the filter obtained from the needle method and the step-by-step insertion of layers.

a refinement technique. We tried to overcome the fact discussed above that the restrictions on variations of the thicknesses for global search have been chosen too narrowly. This method did not yield better results than others because the local minima defined by the narrow-band reflectors were obviously too deep. The result, Fig. 6, is an illustration that the solution for the given case study could not be constructed simply by following the described procedure.

\section{Discussion and Conclusions}

An overview of the results, which illustrates and compares the final values obtained by different design strategies described in Section 2, is given in Table 1. The profiles of the refractive indices for all five solutions are given in Fig. 7. The metric thicknesses are expressed in micrometers. Following the design strategies above, we tried to determine which approach from the chosen synthesis and refinement

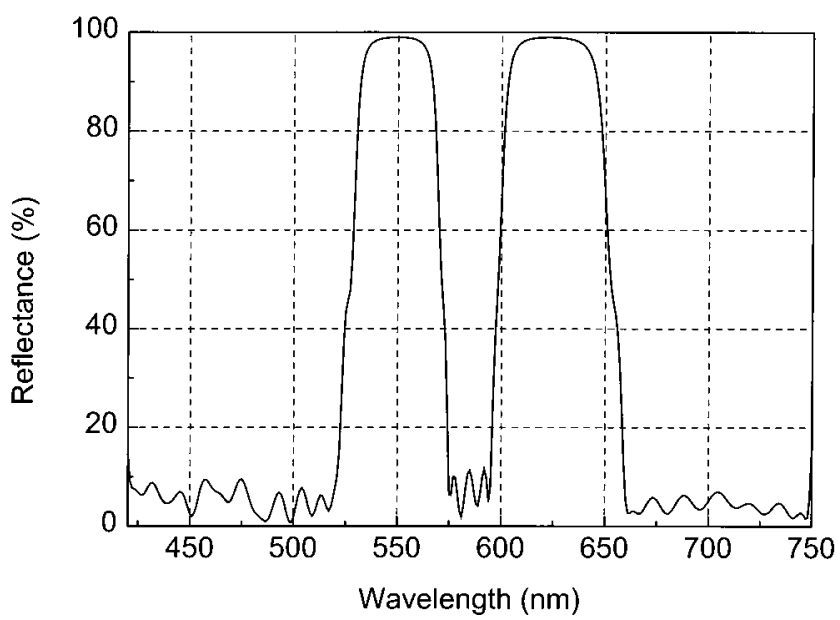

Fig. 5. Reflectance versus wavelength $(\mathrm{nm})$ for the filter obtained by use of the composition of two notch filters created by the needle method. 


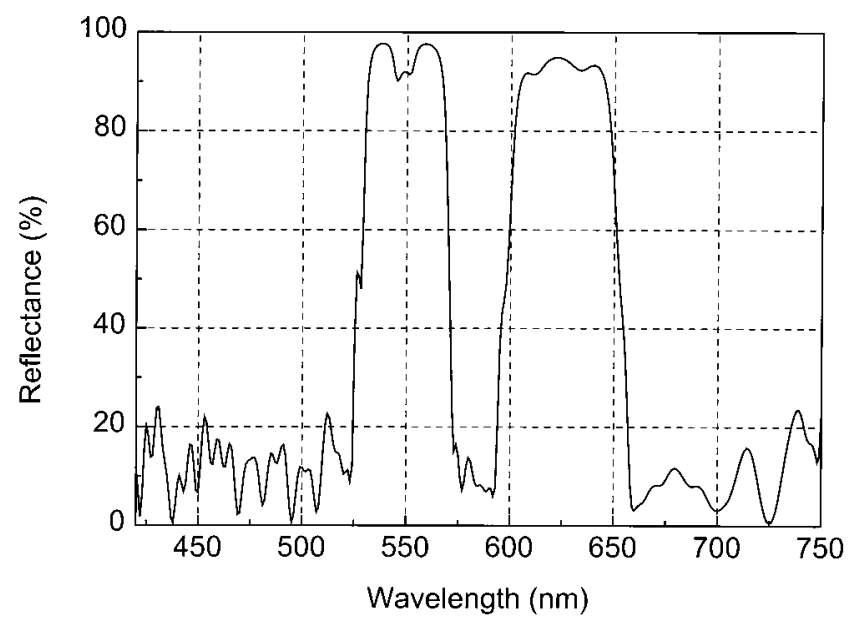

Fig. 6. Reflectance versus wavelength (nanometers) for the filter obtained from a composition of two (L3H $)^{n}$-type filters.

method combinations gave the best results. The best result, in the sense of deviation from the target value, was obtained by a global search followed by variable metrics refinement. Whether this solution should really be considered to be the best is unclear. Comparing the global-based designs with the needlebased designs, we can see that they all have similar deviations and total thicknesses. Which results are the best is really not easy to answer. However, the needle design implemented in Tfcalc 3.2 does not give a superior solution. Maybe this is a possible answer to a question of Tikhonravov et al., ${ }^{6}$ namely, this is a type of problem in which other methods give better results even with a lower number of layers.

Two different types of target function have been used for the design. The continuous target function, where requirements (A)-(E) were entered as five continuous optimization targets, yielded generally better results, although the discrete target function has been well defined in 226 points. On the other hand, the use of a discrete target function enabled a faster approach to the solution. Sometimes a combined use of these two target functions decreases the design time. This method has not been applied here, since the intention was to distinguish one from the other in order to come to reasonable conclusions.

Table 1. Comparison of Final Results from Different Design Procedures

\begin{tabular}{lccc}
\hline \multicolumn{1}{c}{ Synthesis Procedure } & Deviation & $\begin{array}{c}\text { Number } \\
\text { of } \\
\text { Layers }\end{array}$ & $\begin{array}{c}\text { Total } \\
\text { Thickness } \\
\text { (nm) }\end{array}$ \\
\hline $\begin{array}{l}\text { Global search } \\
\text { Modified global search }\end{array}$ & 8.065 & 48 & 6044 \\
$\begin{array}{l}\text { Needle design- } \\
\quad \text { simultaneous }\end{array}$ & 8.198 & 48 & 5851 \\
$\begin{array}{l}\text { Needle design-one by one } \\
\text { Composition of two needles }\end{array}$ & 8.118 & 49 & 5807 \\
$\begin{array}{l}\text { Composition of two narrow- } \\
\text { band reflectors }\end{array}$ & 9.454 & 49 & 6227 \\
\hline
\end{tabular}
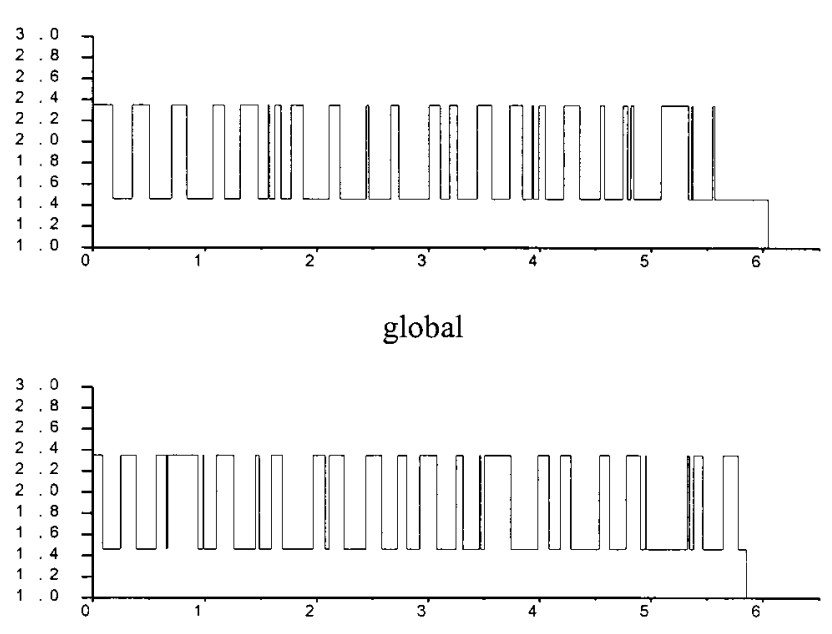

$$
\text { global - modified }
$$
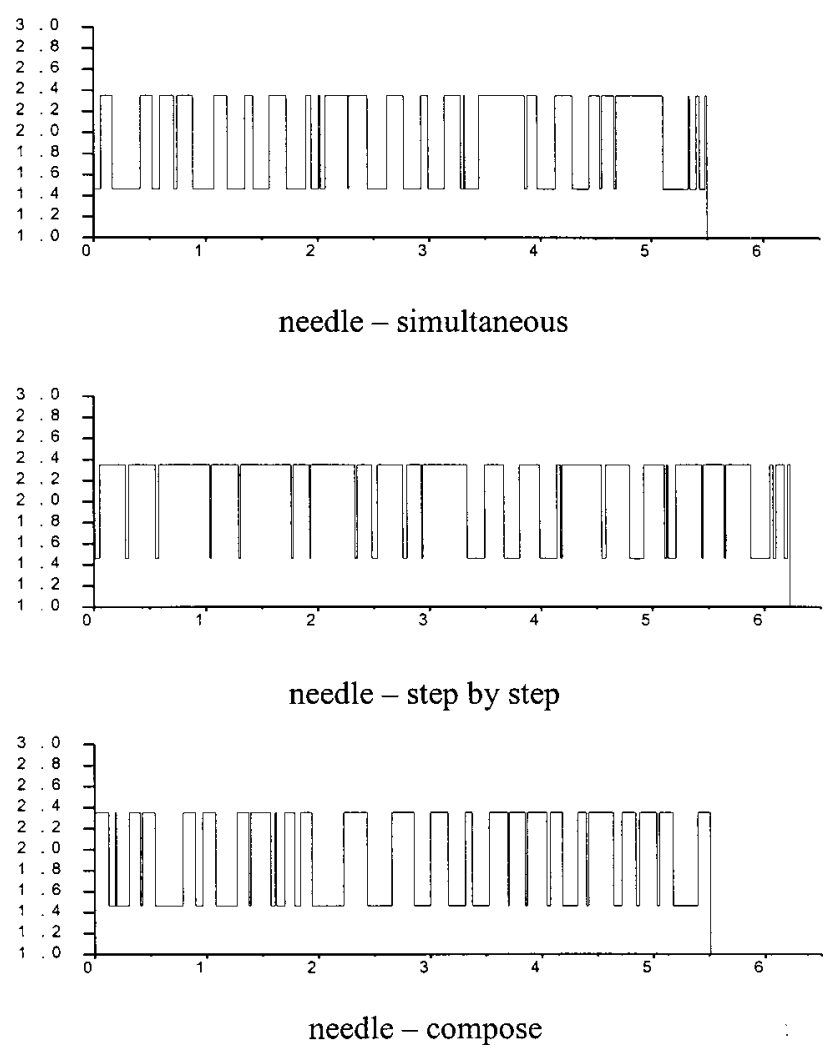

Fig. 7. Refractive-index profile of the five designs ( $n$ versus micrometers).

The strengths and the weaknesses of the software used are reflected partially in the results of this paper. We attempted to avoid erroneous conclusions that might have been generated by the software itself.

\section{Summary}

In this paper different design procedures, in which a given study is used, have been analyzed. The weaknesses and the strengths of each for the given problem have been discussed. It has been found that 
they all lead to more or less similar solutions, i.e., deviation values, but they are all different in the physical sense. Determining the absolute best answer is not possible, since it depends on many factors and the specific needs and requirements of the application. However, it is possible to conclude that the global search and needle methods contain synthesis methods that safely lead to excellent solutions. It is not possible to conclude generally that they will always work in a similar way, but in this study they both yielded similar results. The success of one method or some other method depends also on the fine structure of the software used, i.e., the algorithms that support the method itself. Therefore the multilayer design, except in its practical use, will present a permanent challenge for applied mathematics and physics.

\section{References}

1. J. A. Dobrowolski and R. A. Kemp, "Refinement of optical multilayer systems with different optimization procedures," Appl. Opt. 29, 2876-2893 (1990).
2. J. A. Dobrowolski, "Completely automatic synthesis of optical thin film systems," Appl. Opt. 4, 937-946 (1965).

3. J. A. Dobrowolski, "Versatile computer program for absorbing optical thin film systems," Appl. Opt. 20, 74-81 (1981).

4. S. H. Furman and A. V. Tikhonravov, Optics of Multilayer Systems (Editions Frontieres, Gif-sur-Yvette Cedex, France, 1992), p. 130.

5. P. Baumeister, "Starting designs for the computer optimization of optical coatings," Appl. Opt. 34, 4835-4843 (1995).

6. A. V. Tikhonravov, M. K. Trubetskov, and G. W. DeBell, “Application of the needle optimization technique to the design of optical coatings," Appl. Opt. 35, 5493-5506 (1996).

7. B. T. Sullivan and J. A. Dobrowolski, "Implementation of a numerical needle method for thin-film design," Appl. Opt. 35, 5484-5492 (1996).

8. W. H. Southwell, "Scaling rules for quintic refractive index matching semi-infinite antireflection coating," in Optical Thin Films V: New Developments, R. L. Hall, ed., Proc. SPIE 3133, 65-70 (1997).

9. TFCalc, 1985-1997 Software Spectra, Inc., 14025 N.W. Harvest Lane, Portland, Oreg. 97229.

10. A. V. Tikhonravov, "Needle optimization technique: the history and the future," in Optical Thin Films V: New Developments, R. L. Hall, ed., Proc. SPIE 3133, 2-7 (1997).

11. H. A. Macleod, Thin Film Optical Filters (Adam Hilger, London, 1969), pp. 172-184. 